\title{
Characterization of the Pathogenesis Mechanism after Pseudomonas aeruginosa Infection through Food Consumption Using Chick Embryo Model
}

\author{
Jinsoo Song, Eun-Jung Jin, and Kyoung-Hee Choi ${ }^{1 *}$ \\ Department of Biological Sciences, College of Natural Sciences, Wonkwang University, Iksan 570-749, Korea \\ ${ }^{I}$ Department of Oral Microbiology, College of Dentistry, Wonkwang University, Iksan 570-749, Korea
}

\begin{abstract}
This study introduced a chick embryos' infection model to elucidate the pathogenesis mechanism of Pseudomonas aeruginosa, which causes serious diseases in human after ingestion of $P$. aeruginosa-contaminated animal originated foods. The embryonic chick model is able to give a rapid and relatively inexpensive method to assess bacterial pathogenicity compared to embryos of other vertebrates. Embryos were infected with $P$. aeruginosa and elastase-deficient $P$. aeruginosa. After infection with $P$. aeruginosa cells, total bacterial cell numbers and gelatinase activities in the embryos were compared. Thereafter, precartilage condensation and chondrogenesis were assessed by peanut agglutinin (PNA) binding on day 3 and by Alcian blue staining for sulfated proteoglycans on day 5, respectively. P. aeruginosa significantly increased in embryos, resulting in abnormal limb development, whereas $P$. aeruginosa defective in elastase activity partly impaired proliferation. In addition, $P$. aeruginosa-infected chick embryos significantly stimulated the production of matrix metalloproteinases. Several analyses showed that elevated proteases suppressed the proliferation and survival of chondrogenic cells. The results show that this infection model was a useful assay to determine the virulence mechanism of $P$. aeruginosa in human after intake of microbiologically contaminated foods.
\end{abstract}

Key words: Pseudomonas aeruginosa, chick embryos, elastase, pathogenesis

\section{Introduction}

Bacterial infection in humans arises frequently after ingestion of contaminated foods with various pathogens, especially from pork, beef, poultry meat, and eggs (Namata et al., 2009). To escape from the prevalence of bacterial pathogens risking human health, it is necessarily preceded to undertake the problem at the level of livestock farm, thus diminishing the cross-contamination within a herd or flock (Collard et al., 2007; Namata et al., 2005). Although animals are infected with pathogenic bacteria, they become often asymptomatic, but can be spread readily at the farm (EFSA (European Food Safety Authority), 2007; Namata et al., 2009). In addition to horizontal transmissions of pathogens, bacterial infection can be vertically transmitted from mother to fetus, which is

\footnotetext{
*Corresponding author: Kyoung-Hee Choi, Department of Oral Microbiology, College of Dentistry, Wonkwang University, Iksan 570-749, Korea. Tel: 82-63-850-6911, Fax: 82-63-8507313, E-mail: kheechoi@wku.ac.kr
}

one of the important routes in the contamination of flocks with pathogenic bacteria (Namata et al., 2009).

According to previous studies, the primary genera found in pasteurized egg products are Alicaligenes, Bacillus, Escherichia, Proteus, Pseudomonas, and Gram-positive bacteria (Schmidt-Lorenz, 1983; Cunningham, 1995). Among them, psychrotrophs including the genera Pseudomonas primarily cause spoilage of egg whites at refrigerated condition (MacKenzie and Skerman, 1982). Thus, the shelf-life of liquid eggs is generally short at refrigeration temperatures.

Among Pseudomonas species, Pseudomonas aeruginosa is one of the most significant food spoilage organisms and a ubiquitous, opportunistic human pathogen which is able to cause life-threatening infections in injured, burned, and immunocompromised patients (Myszka and Czaczyk, 2009; Van Delden and Iglewski, 1998). P. aeruginosa causes off-flavor in various foods including meats, vegetables, and fish (Bower et al., 1996). Especially, $P$. aeruginosa is known to dominate proteinaceous foods including meat, poultry, milk, and fish stored at chill tem- 
peratures (Gram et al., 2002). Also, ingestion of $P$. aeruginosa-contaminated foods may result in various diseases such as urinary tract infections, respiratory system infections, dermatitis, soft tissue infections, and bacteremia in humans using various virulence-related factors. Among a number of virulence factors of $P$. aeruginosa, several determinants are required for causing disease in diverse hosts, but others only in specific host species. In addition, since $P$. aeruginosa is an agent of various diseases, evolutionarily divergent host infection models were developed and applied to characterize the factors and virulence mechanism of $P$. aeruginosa relevant to clinical settings.

Among them, genetically accessible invertebrates such as Caenorhabditis elegans (roundworm), Drosophila melanogaster (fruitfly), and Dictyostelium discoideum (amoeba) have been developed to learn $P$. aeruginosa pathogenesis (D'Argenio et al., 2001; Mahajan-Mihlos et al., 1999; Pukatzki et al., 2002). Unlike rodent models, these model hosts possess several advantages which include costeffectiveness, small size and short life cycle of the organisms, thus enabling tests such as genome-wide genetic screens (Clatworthy et al., 2009). However, the use of the invertebrate hosts is unfeasible because vertebrate immune responses are distinguished from invertebrate ones, such that the latter is not related to adaptive immunity, one of the features of humans (Clatworthy et al., 2009).

A chick embryo, which is vulnerable to infection with many pathogenic bacteria, is one of critical vehicles to conduct mother-to-child transmission of bacterial infection. The embryonic chick model is capable of giving a rapid and relatively inexpensive measure of the toxicity of a number of pathogens. Chick embryos are relatively inexpensive and easy to maintain in a laboratory setting as compared with embryos of other vertebrates. Unlike other higher vertebrate systems, such as mouse ova, fertilized eggs can be sustained in a simple incubator, with need for upkeep or any manipulation of the mother; therefore expenditures of time and money for feeding and cage cleaning are unnecessary. Chicks are also ideal for developmental studies because the embryo is easily accessible and relatively easy to manipulate. Penetration of the egg and access to the developing embryo require much less time and effort than in other vertebrates. In mammals, access to the embryo cannot be acquired without manipulation of the mother, which is generally an invasive procedure that is stressful and potentially dangerous for both the mother and the developing embryo. Thus, the chicken embryo provides an excellent model for the study of the virulence of pathogens for humans, such as Neisseria gonorrhoeae and Neisseria meningitidis (Buddingh, 1970; Bumgarner and Finkelstein, 1973; Diena et al., 1975; Frasch et al., 1976). Chick embryos have been used to test host-related bacterial virulence and pathology in a wide variety of bacteria such as Francisella spp., Mycoplasma lipofaciens, N. gonorrhoeae, N. meningitidis, Streptococcus flexneri, Escherichia coli and Vibrio cholerae (Lierz and Hafez, 2008; Nix et al., 2006; Payne and Finkelstein, 1978). The use of embryonated hens' eggs was performed also in $P$. aeruginosa infection to screen antibacterial therapeutic substances as an alternative in vivo model (Hartl et al., 1997). Up to date, however, it has not been utilized to define important molecular mechanisms regarding virulence of $P$. aeruginosa in eggs.

Therefore, our objective in this work was to develop a useful assay system that tests the virulence and pathogenesis mechanism of $P$. aeruginosa, and elucidates specific features playing a major role in a human-pathogen interaction. This further will suggest an effective way to control food spoilage and vertical transmission of bacterial infection, ultimately contributing to human health.

\section{Materials and Methods}

\section{Bacterial strains and growth conditions}

Escherichia coli DH5 $\alpha$ and P. aeruginosa PA14 strains (including wild-type, las $B$ and $g f p$ mutants) were maintained on Luria-Bertani medium (LB; 10 g per liter tryptone, $5 \mathrm{~g}$ per liter yeast extract, $10 \mathrm{~g}$ per liter $\mathrm{NaCl}$; Difco) at $37^{\circ} \mathrm{C}$ incubator. $P$. aeruginosa PA14 strain containing a chromosomal copy of green fluorescence protein $(g f p)$ was created using mini-Tn7 gene integration system as previously described (Choi and Schweizer, 2006).

\section{Bacterial inoculation into chicken embryos}

Specific pathogen-free fertile eggs from White Leghorn chickens fed antibiotic-free meals were incubated in a Humidaire incubator (Saesil, Gyeonggi-do, Korea) with automatic turning, controlled temperature (37.3 to 37.8 ${ }^{\circ} \mathrm{C}$ ), humidity (50 to $55 \%$ ), and air circulation. The age of the embryos was determined morphologically by using the Hamburger series of normal stages (Hamburger and Hamilton, 1951) in the chicken embryo, with a minimum of three eggs per experiment sacrificed for this purpose.

Nine day-old chick embryos were selected for inoculation. The eggs were opened by making a window over the chorioallantoic membrane and allowing the membrane to recede. They were inoculated with a sublethal infection dose, $10^{4} \mathrm{CFU}$ of $P$. aeruginosa cells, and the window 
was covered with adhesive tape. At $24 \mathrm{~h}$ after inoculation, suspensions of embryos, $20 \%$ by weight, were made by grinding the embryo in a Waring blendor (Waring blendor, Torrington, CT, USA) in cold saline buffered with phosphate. Such suspensions were centrifuged in the Swedish angle centrifuge at about 3,000 g. for $15 \mathrm{~min}$ before titration of the supernatant.

\section{Bacterial enumeration from infected embryos}

Dead embryos were removed and survived individual chick embryos were collected. The eggs were blended followed by plating dilutions of the homogenized egg extracts on LB plates to enumerate colony forming units (CFU) within infected eggs. Total bacterial numbers were standardized by dividing by each egg weight.

\section{Gelatin zymography}

Gelatinase activity was determined using the gelatin zymography assay with $0.1 \%$ gelatin (Sigma, St. Louis, Mo, USA) as a substrate in 10\% SDS-polyacrylamide gel. Embryo lysates was subjected to SDS-PAGE under non-reducing conditions. After electrophoresis, gels were washed three times with $2.5 \%$ Triton X-100 (Sigma) in water, then incubated with $50 \mathrm{mM}$ Tris buffer, $\mathrm{pH}$ 7.4, containing $0.2 \%$ Brij $35,5 \mathrm{mM} \mathrm{CaCl}_{2}$, and $1 \mathrm{mM} \mathrm{NaCl}$, overnight at $37^{\circ} \mathrm{C}$ in a closed container. Gels were stained for 30 min with $0.25 \%$ Coomassie Blue R-250 (Amresco, Cochran Road Solon, OH, USA ) in 10\% acetic acid and $45 \%$ methanol, and destained for $30 \mathrm{~min}$ using a mixture of $20 \%$ acetic acid, $20 \%$ methanol and $17 \%$ ethanol. Areas of gelatinase activity were detected as clear bands against the blue-stained gelatin background.

\section{Cell culture and cell proliferation assay}

For limb mesenchymal cell culture, the modified technique of Ahrens et al. (1993) was used. Briefly, micromass cultures used $10 \mu \mathrm{L}$ drops of cells at $10^{7}$ cells $/ \mathrm{mL}$ maintained in Ham's F-12 medium (Gibco Invitrogen, Grand Island, NY, USA) containing 10\% FBS. Micromass cultures were also incubated with or without purified gelatinase induced by inoculation. Gelatinase concentration was achieved by ultrafiltering the sample solution through Ultracel YM-30 membrane (Millipore, Concord Road, Billerica, MA, USA).

Proliferation of mesenchymal cells was determined by direct counting of cells from micromass cultures. Control and treated cultures were maintained for the indicated number of days, detached with trypsin/EDTA (Gibco, Invitrogen) solution and counted in triplicate using a hema- cytometer (Superior, Lauda-Konigshofen, Germany).

\section{Analysis of cell differentiation}

Chondrogenesis was measured by Alcian blue (Sigma) staining of sulfated cartilage glycosaminoglycans. Alcian blue-bound sulfated glycosaminoglycans were extracted with $6 \mathrm{M}$ guanidine- $\mathrm{HCl}$ (Sigma), and quantified by measuring the absorbance of the extracts at $600 \mathrm{~nm}$ by spectrophotometer (Tecan, Männedorf, Switzerland).

\section{Results}

\section{Morphological defect during developmental pro- gression of chicken embryos after infection with $P$. aeruginosa}

Nine day-old chick embryos were infected with various doses of wild-type $P$. aeruginosa and E. coli strain (EC) ranging from $10^{2}$ to $10^{7} \mathrm{CFU}$ at an interval of a logarithmic dilution. As a result, embryonated eggs were more fatal to $P$. aeruginosa than to E. coli, and unlike E. coli, $10^{4} \mathrm{CFU}$ of $P$. aeruginosa cells was shown as a sublethal dose in embryonated eggs (data not shown). At the infection dose, chick embryos were survived at $24 \mathrm{~h}$ after infection, while they became dead over $24 \mathrm{~h}$ incubation (data not shown). Therefore, chick embryos were incubated with an inoculation of $10^{4} \mathrm{CFU}$ of bacterial cells for $24 \mathrm{~h}$ in order to observe a severe and clear effect of bacterial pathogenesis including morphological defect during developmental progression of embryonated eggs. Wildtype $P$. aeruginosa strain survived and proliferated fourfold more than nonpathogenic $E$. coli strain within chick embryos (Fig. 1A). The bacterial infectivity was visualized with parafilm sectioning and gram staining of chicken head and body infected with $P$. aeruginosa cells (Fig. 1B). P. aeruginosa cells successfully established their growth within both chicken head and body.

Elastase, encoded by lasB gene, is one the most significant invasive factor during $P$. aeruginosa infection (Van Delden, 2004). Therefore, the virulence of wild-type and its las $B$ mutant were compared. Infection rates of $P$. aeruginosa and las $B$ mutant in chicken embryos were compared by recovering the embryos and enumerating bacterial cells (Fig. 1A). As a result, although wild-type and its las $B$ mutant grow at a same rate (data not shown), the las $B$ mutant displayed two-fold decreased infection rate compared to the wild-type $P$. aeruginosa strain. However, the infectivity of the las $B$ mutant was still relatively high compared to one of the E. coli strain. It is thought to be due to a relatively low level of elastolytic activity by 
A

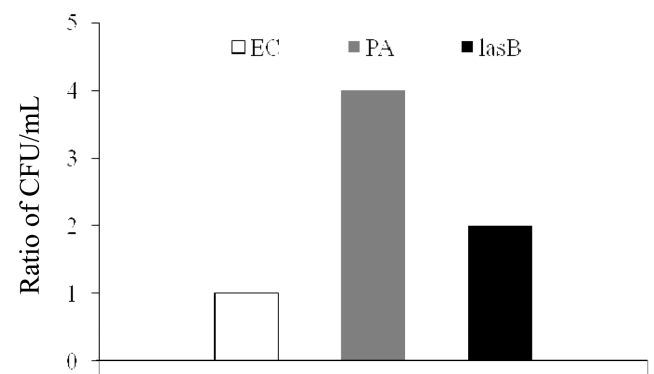

B

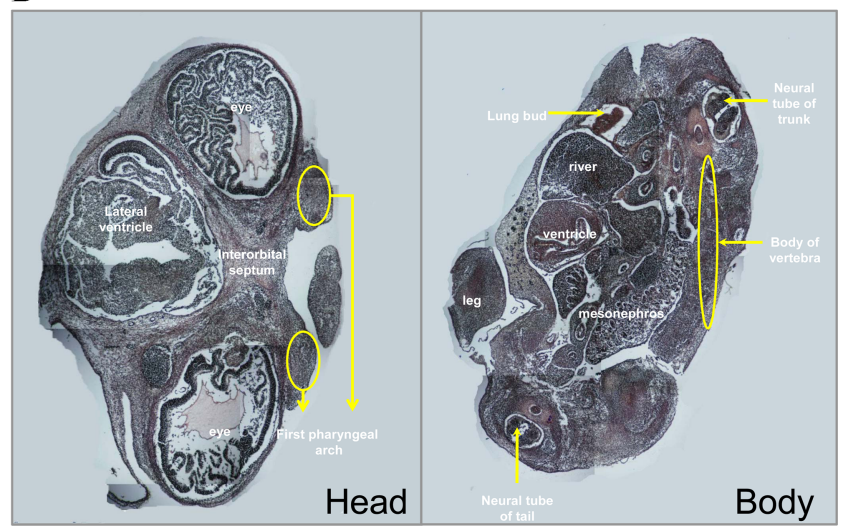

Fig. 1. A. Bacterial enumeration from infected embryos. Chick embryos were infected with $E$. coli DH5a (EC), $P$. aeruginosa (PA), $P$. aeruginosa defected in elastase activity (lasB). Ratio of CFU/mL shown on the Y-axis is calculated by dividing each $\mathrm{CFU} / \mathrm{mL}$ by the one of EC. B. Parafilm section and gram staining of chicken head and body infected with $P$. aeruginosa. Area of $P$. aeruginosa cells was shown in pink colors.

LasA (Kessler and Safrin, 1997).

The malformation of limb also was observed when $\mathrm{HH}$ stage 31 chick embryos were inoculated with $P$. aeruginosa for $24 \mathrm{~h}$, suggesting the defection on the process of endochondral ossification by infection of invasive $P$. aeruginosa (Fig. 2).

\section{$P$. aeruginosa-induced gelatinase stimulates apop- totic death of chondrogenic progenitors}

To determine whether inoculation of chick embryos with $P$. aeruginosa lead to induction of gelatinase, zymography was performed (Fig. 3A). Lysates of P. aeruginosa-inoculated chick embryo showed distinct bands on gelatin gels, indicating the presence of several Matrix metalloproteinase (MMP)s with gelatinolytic and/or collagenolytic activity of $30-50 \mathrm{kDa}$ proteins compared to EC-inoculated embryo. This indicated that this increased activity might be responsible for virulent actions of $P$. aeruginosa including abnormal limb development. According to previous studies, corneas were destructed with infection of $P$. aeruginosa by the mechanisms that $P$. aeruginosa exproteases stimulated expression of MMPs releas-

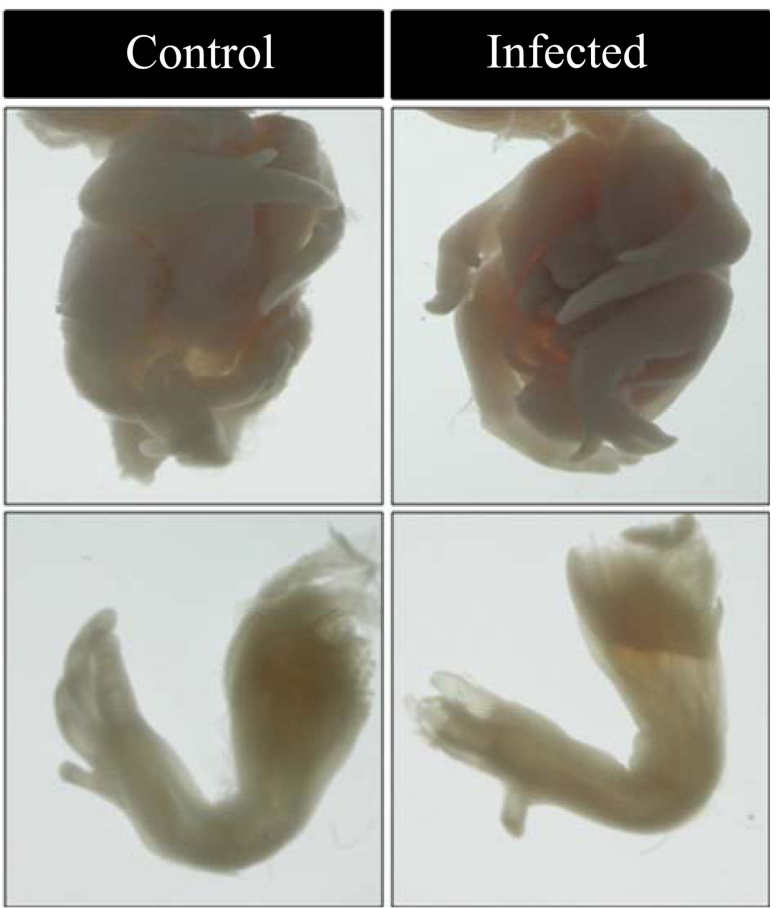

Fig. 2. Morphological defect during developmental progression of chicken embryos after infection with $P$. aeruginosa.

ed by corneal stromal cells and $P$. aeruginosa elastase proteolytically activated MMPs (Miyajima et al., 2001).

To confirm this, proteases activated by inoculation of $P$. aeruginosa were purified and used for determining the effects on chondrogenesis. Chondroblasts isolated from wing buds were cultured at a density of $2 \times 10^{7}$ cells $/ \mathrm{mL}$ and stimulated with purified proteases. Precartilage condensation and chondrogenesis were assessed by peanut agglutinin (PNA) binding on day 3 and by Alcian blue staining for sulfated proteoglycans on day 5 , respectively. With treated of $P$. aeruginosa-induced protease, PNA staining and Alcian blue uptake were significantly decreased (Fig. 3B). These results demonstrate that $P$. aeruginosa-induced protease inhibits sulfated proteoglycan accumulation and cartilage nodule formation during chondrogenesis.

Precartilage condensation is known to be regulated by cell-density due to cell proliferation or apoptotic cell death (Maini and Solursh, 1991; Solursh, 1989). We first examined if $P$. aeruginosa-induced protease-inhibited chondrogenesis was due to regulation of cell proliferation. During chondrogenic differentiation, the cell numbers increased in control cultures. Exposure of cells to P. aeruginosainduced protease suppressed the proliferation of chondrogenic competent cells as determined by direct cell counting (Fig. 3C). These data indicate that the negative 


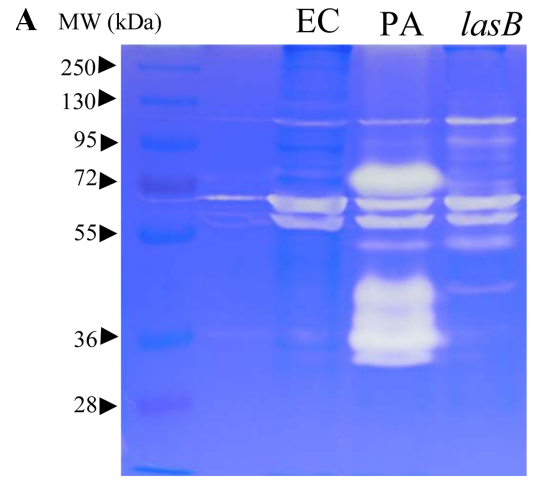

B
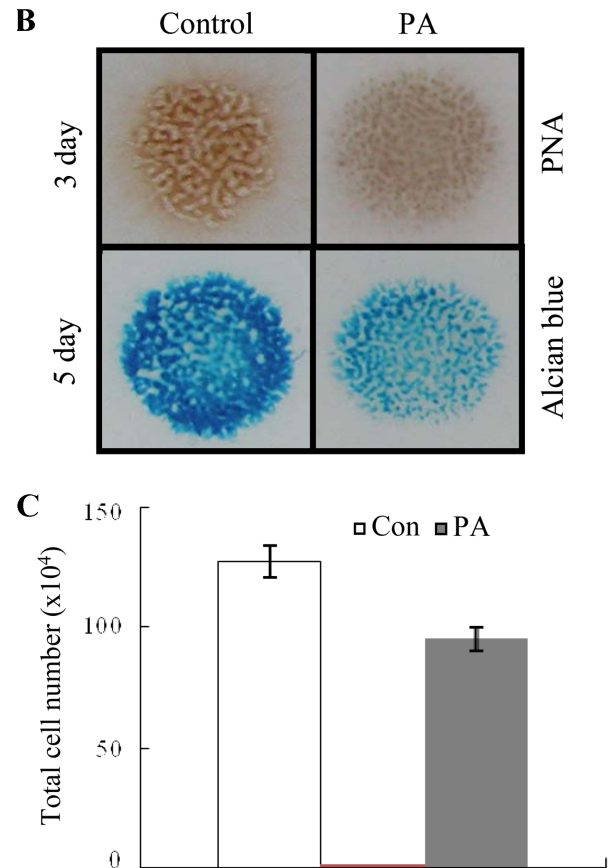

Fig. 3. A. Gelatin zymography. Embryo lysates after infection with E. coli DH5 $\alpha$ (EC), P. aeruginosa (PA), P. aeruginosa defected in elastase activity (lasB) were used for the gelatin zymography. B. Effect of $\boldsymbol{P}$. aeruginosa-induced protease on sulfated proteoglycan accumulation and cartilage nodule formation during chondrogenesis. After exposure of cells to $P$. aeruginosa-induced protease, precartilage condensation and chondrogenesis were assessed by peanut agglutinin (PNA) binding and by Alcian blue staining for sulfated proteoglycans. C. Effect of suppressed chondrogenesis induced by the protease on the proliferation and survival of chondrogenic competent cells by direct cell counting.

regulatory action by $P$. aeruginosa-induced protease results by modulating the proliferation and survival of chondrogenic competent cells.

\section{Discussion}

In this study, we show that chick embryos can be utilized as an infection host for elucidating the mechanism of bacteria-mediated pathogenesis. So far, its use has been limited to test bacterial pathogenicity and to screen antibacterial drugs in various bacteria (Lierz and Haffez, 2008; Nix et al., 2006; Payne and Finkelstein, 1978; Hartl et al., 1997). However, a usefulness of its application successfully expanded into the characterization of central molecular mechanisms regarding bacterial virulence in the present study.

$P$. aeruginosa cells successfully survived and proliferated in chicken head and body for $24 \mathrm{~h}$ after inoculation of bacterial cells into chick embryos. The infectivity of the las $B$ mutant was relatively low compared to one of the wild-type $P$. aeruginosa strain. During $P$. aeruginosa infection, proteases play a major role in virulence and tissue penetration (Tang et al., 1996; Twinning et al., 1993). $P$. aeruginosa synthesizes several proteases, including LasA and LasB elastases, and alkaline protease. Elastin present in all invertebrates is a principal protein of connective tissues and its degradation is carried out by the LasB enzyme, which is known to be an important enzyme with elastolytic activity (Van Delden, 2004; Kessler and Safrin, 1997). Particulary, LasB elastase degrades not only elastin but also collagen, which is another major component of connective tissues (Heck et al., 1986). Elastases were determined as a main virulence determinant of causing the pulmonary hemorrhages during invasive P. aeruginosa clinical settings (Van Delden, 2004). Cowell et al (2003) showed that mutation of lasB gene encoding LasB enzyme suppressed $P$. aeruginosa invasion. In addition, previous studies demonstrated that $P$. aeruginosa las $B$ mutant exhibited decreased virulence in animal models (Tang et al., 1996; Tamura et al., 1992).

In addition, chick embryos suffered the morphological defect on limb during developmental progression of chicken embryos after infection with $P$. aeruginosa. It is suggested that the malformation of chicken limb resulted from the invasiveness of the bacteria by elastolytic action on embryonic connective tissues. However, how it mediates the morphological defect during limb formation is not clear at this time. At the molecular level, P. aeruginosa cells significantly stimulated the production of several matrix metalloproteinases, indicating the possible role in bacterial virulence such as deformed limb development. Considerably increased matrix metalloproteases played a negative role in sulfated proteoglycan accumulation and cartilage nodule formation during chondrogenesis through the down-regulation of chondrogenic cell proliferation. However, a better and complete understanding of the bacteria-mediated pathogenic mechanism should 
be accomplished in future work.

Taken together, chick embryo infection model was very useful to establish the bacterial virulence mechanism by examining the morphological effect and the molecular change via host-bacteria interaction. In addition, the study will provide an idea to prevent food spoilage and lifethreatening disease in human by controlling bacterial infection and spread.

\section{Acknowledgement}

This study was supported by a grant of the Korea Healthcare technology R\&D Project, Ministry for Health, Welfare \& Family Affairs, Republic of Korea (A084717).

\section{References}

1. Ahrens, M., Ankenbauer, T., Schröder, D., Hollnagel, A., Mayer, H., and Gross, G. (1993) Expression of human bone morphogenetic proteins-2 or -4 in murine mesenchymal progenitor $\mathrm{C} 3 \mathrm{H} 10 \mathrm{~T} 1 / 2$ cells induces differentiation into distinct mesenchymal cell lineages. DNA Cell. Biol. 12, 871-880.

2. Bower, C. K., McGuire, J., and Daeschel, M. A. (1996) The adhesion and detachment of bacteria and spores on food-contact surfaces. Trends Food Sci. Tech. 7, 152-157.

3. Buddingh, G. J. (1970) The chick embryo for the study of infection and immunity. J. Infect. Dis. 121, 660-663.

4. Bumgarner, L. R. and Finkelstein, R. A. (1973) Pathogenesis and immunology of experimental gonococcal infection: virulence of colony types of Neisseria gonorrhoeae for chicken embryos. Infect. Immun. 8, 919-924.

5. Choi, K. H. and Schweizer, H. P. (2006) mini-Tn7 insertion in bacteria with single att $\mathrm{Tn} 7$ sites: example Pseudomonas aeruginosa. Nat. Prot. 1, 153-161.

6. Clatworthy, A. E., Lee, J. S., Leibman, M., Kostun, Z., Davidson, A. J., and Hung, D. T. (2009) Pseudomonas aeruginosa infection of zebrafish involves both host and pathogen determinants. Infect. Immun. 77, 1293-1303.

7. Collard, J. M., Bertrand, S., Dierick, K., Godard, C., Wildemauwe, C., Vermeersch, K., Duculot, J., Van Immerseel, F., Pasmans, F., Imberechts, H., and Quinet, C. (2007) Drastic decrease of Salmonella Enteritidis isolated from humans in Belgium in 2005, shift in phage types and influence on foodborne outbreaks. Epidemiol. Infect. 136, 771-781.

8. Cowell, B. A., Twining, S. S., Jeffrey, A. H., Kwong, M. S. F., and Fleiszig, S. M. J. (2003) Mutation of las $A$ and las $B$ reduces Pseudomonas aeruginosa invasion of epithelial cells. Microbiol. 149, 2291-2299.

9. Cunningham, F. E. (1995) Egg-product pasteurization. In: Egg science and technology. Stadelman, W. J. and Cotterill, O. J. (eds) Food Products Press, NY, 289-322.

10. D'Argenio, D. A., Gallagher, L. A., Berg, C. A., and Manoli, C. (2001) Drosophila as a model host for Pseudomonas aeruginosa infection. J. Bacteriol. 183, 1466-1471.
11. Diena, B. B, Lavergne, G., Ryan, A., Ashton, F. E., Wallace, R., and Perry, M. (1975) The chick embryo in studies of virulence and immunity with Neisseria gonorrhoeae. Rev. Can. Biol. 34, 213-220.

12. EFSA (European Food Safety Authority) (2007) Salmonella. The community summary report on trends and sources of zoonoses, zoonotic agents, antimicrobial resistance and foodborne outbreaks in the European Union in 2005. 27-81.

13. Frasch, C. E., Parkes, L., MeNelis, R. M., and Gotschlick, E. C. (1976) Protection against group B meningococcal disease. I. Comparison of group-specific and type-specific protection in the chick embryo model. J. Exp. Med. 1144, 319-329.

14. Gram, L., Ravn, L., Rasch, M., Bruhn, J. B., Christensen, A. B., and Givskov, M. (2002) Food spoilage-interactions between food spoilage bacteria. Int. J. Food Microbiol.78, 79-97.

15. Hamburger, V. and Hamilton, H. L. (1951) A series of normal stages in the development of the chick embryo. J. Morphol. 88, 49-92.

16. Hartl, A., Mollmann, U., Schrinner, E., and Stelzner, A. (1997) Pseudomonas aeruginosa in embryonated hen's eggs. An alternatives in vivo model for the screening of antibacterial substances. Arzneimittelforschung 47, 1061-1064.

17. Heck, L. W., Morihara, K., McRae, W. B., and Miller, E. J. (1986) Specific cleavage of human type III and IV collagens by Pseudomonas aeruginosa elastase. Infect. Immun. 51, 115-118.

18. Kessler, E. and Safrin, M. (1997) Inhibitors and specificity of Pseudomonas aeruginosa LasA. J. Biol. Chem. 272, 98849889.

19. Lierz, M., and Hafez, H. M. (2008) Time-dependent recovery of Mycoplasma lipofaciens (strain ML64) from incubated infertile chicken eggs and dead in shell chicken embryos. Avian Dis. 52, 441-443.

20. Mackenzie, K. A. and Skerman, V. B. D. (1982) Microbial spoilage in unpasteurised liquid whole egg. Food Technol. Aust. 34, 524-528.

21. Mahajan-Miklos, S., Tan, M. W., Rahme, L. G., and Ausubel, F. M. (1999) Molecular mechanisms of bacterial virulence elucidated using a Pseudomonas aeruginosa-Caenorhabditis elegans pathogenesis model. Cell 96, 47-56.

22. Maini, P. K. and Solursh, M. (1991) Cellular mechanisms of pattern formation in the developing limb. Int. Rev. Cytol. 129, 91-133.

23. Miyajima, S., Akaike, T., Matsumoto, K., Okamoto, T., Yoshitake, J., Hayashida K, Negi, A., and Maeda, H. (2001) Matrix metalloproteinases induction by pseudomonal virulence factors and inflammatory cytokines in vitro. Microb. Pathog. 31, 271-281.

24. Namata, H., Welby, S., Aerts, M., Faes, C., Abrahantes, J. C., Imberechts, H., Vermeersch, K., Hooyberghs, J., Méroc, E., and Mintiens, K. (2009) Identification of risk factors for the prevalence and persistence of Salmonella in Belgian broiler chicken flocks. Prev. Vet. Med. 90, 211-222.

25. Nix, E. B., Cheung, K. K. M., Wang, D., Zhang, N., Burke, R. D., and Nano, F. E. (2006) Virulence of Francisella spp. Chicken embryos. Infect. Immun. 74, 4809-4816. 
26. Payne, S. M., and Finkelstein, R. A. (1978) The critical role of iron in host-bacterial interactions. J. Clin. Invest. 61, 1428-1440.

27. Pukatzki, S., Kessin, R. H., and Mekalanos, J. J. (2002) The human pathogen Pseudomonas aeruginosa utilizes conserved virulence pathways to infect the social amoeba Dictyostelium discoideum. Proc. Natl. Acad. Sci. USA 99, 31593164.

28. Schmidt-Lorenz, W. (1983) Collection of methods for the microbiological examination of foods. Verlag Chemie, Weinheim, Germany, 15.1-15.22

29. Solursh, M. (1989) Differentiation of cartilage and bone. Curr. Opin. Cell. Biol. 1, 989-994.

30. Tamura, Y., Suzuki, S., and Sawada, T. (1992) Role of elastase as a virulence factor in experimental Pseudomonas aeruginosa infection in mice. Microb. Pathog. 12, 237-244.

31. Tang, H. B., Dimango, E., Brian, M. J., Gambello, M. J.,
Iglewski, B. J., Goldberg JB, and Prince, A. (1996) Contribution of specific Pseudomonas aeruginosa virulence factors to pathogenesis of pneumonia in a neonatal mouse model of infection. Infect. Immun. 64, 37-43.

32. Twinning, S. S., Kirschner, S. E., Mahnke, L. A., and Franke, D. W. (1993) Effect of Pseudomonas aeruginosa elastase, alkaline protease, and exotoxin A on corneal proteinases and proteins. Invest. Ophthalmol. Vis. Sci. 34, 2699-2712.

33. Van Delden, C. V. (2004) Virulence factors in Pseudomonas aeruginosa. In: Pseudomonas: virulence and gene regulation. Kluwer Academic/Plenum Publishers, New York, pp. 23-45.

34. Van Delden, C., and Iglewski, B. H. (1998) Cell-to-cell signaling and Pseudomonas aeruginosa infections. Emerg. Infect. Dis. 4, 551-560.

(Received 2010.4.22/Revised 2010.5.27/Accepted 2010.5.28) 